\title{
Hematomas de cerebelo como complicación de drenaje lumbar. Presentación de dos casos y revisión de la literatura
}

L.M. Bernal-García; J.M. Cabezudo-Artero; M. Ortega-Martínez; I. Fernández-Portales; L.F. Ugarriza-Echebarrieta; M. Pineda-Palomo; L.F. Porras-Estrada y L.F. Gómez-Perals

Servicio de Neurocirugía. Hospital Universitario Infanta Cristina. Badajoz.

\section{Resumen}

Los hematomas de cerebelo a distancia (HCD) son una complicación rara, consistente en la aparición de un hematoma en el cerebelo tras intervenciones quirúrgicas en áreas que no guardan relación anatómica con él. Esta complicación ha sido descrita tras cirugía supratentorial $\mathrm{y}$, menos frecuentemente, tras cirugía raquídea con apertura dural con importante pérdida de líquido cefalorraquídeo (LCR).

Presentamos dos pacientes con un hematoma de cerebelo tras la colocación de un drenaje lumbar externo (DLE) por sospecha diagnóstica de hidrocefalia crónica del adulto (HCA). Ambos evolucionaron favorablemente tras la retirada de los DLE. Así mismo, realizamos una revisión de la literatura sobre los mecanismos etiopatogénicos, diagnóstico, tratamiento y pronóstico de los HCD.

PALABRAS CLAVE: Drenaje lumbar. Hematoma de cerebelo. Hematomas a distancia. Hidrocefalia crónica del adulto. Hidrocefalia normotensiva.

Remote cerebellar hemorrhage after lumbar spinal fluid drainage. Report of two cases and literature review

\section{Summary}

Remote cerebellar hematoma, a cerebellar hematoma occurring after performing a surgical procedure in an anatomical distant area from the cerebellum, is a rare complication. It has been reported after supratentorial surgery and, less often, after spinal surgery with dural opening with important loss of cerebrospinal fluid. We report the occurrence of remote cerebellar hemorrhage after lumbar spinal fluid drainage in two patients with suspected normal pressure hydrocephalus. They were managed conservatively with good outcome. We review the pathologic mechanism, diagnostic procedures,

Recibido: 20-08-07. Aceptado: 20-09-07 management and prognosis of remote cerebellar hemorrhage.

KEY WORDS: Lumbar spinal fluid drainage. Cerebellar hemorrhage. Remote hemorrhage. Chronic hydrocephalus. Normal pressure hydrocephalus.

Introducción

El drenaje lumbar externo (DLE) es un procedimiento comúnmente utilizado en protocolos de diagnóstico de pacientes con sospecha de hidrocefalia crónica del adulto (HCA). También se utiliza en el tratamiento de fístulas de LCR y como paso previo a otros procedimientos intracraneales para conseguir una adecuada relajación del cerebro.

Esta es una técnica sencilla, pero no está exenta de complicaciones. La más frecuente es la aparición de un síndrome de hipotensión de $\mathrm{LCR}^{24.28}$. Sin embargo se han descrito otras más graves, como infecciones ${ }^{1,20,28}$, neumoencéfalo ${ }^{1,14}$, parálisis de cuerda vocal ${ }^{28}$, infarto cerebral ${ }^{28}$, hemorragias cerebrales múltiples $^{29}$ o herniación transtentorial ${ }^{3,32}$. A su vez los HCD han sido descritos como una complicación rara tras cirugía supratentorial $1^{4,6,8,10,12,16-18,22,23,27,31,34,35,36-38}$ y, menos frecuentemente tras cirugía raquídea con apertura dural $^{2,7,9,11,13,19,21,25,26,30,33}$, aparentemente en relación con la pérdida de LCR.

Presentamos, en este trabajo, una nueva complicación de los DLE, consistente en la aparición de un hematoma de cerebelo, de la que no hemos encontrado casos similares en nuestra revisión bibliográfica. Se discuten los posibles mecanismos etiopatogénicos y aspectos sobre el diagnóstico, tratamiento y pronóstico de los HCD

Abreviaturas. DLE: drenaje lumbar externo. DVE: drenaje ventricular externo. HCA: hidrocefalia crónica del adulto. HCD: hematoma de cerebelo a distancia. HDL: hernia discal lumbar. HTA: hipertensión arterial. LCR: líquido cefalorraquideo. RM: resonancia magnética. TC: tomografia computarizada. 


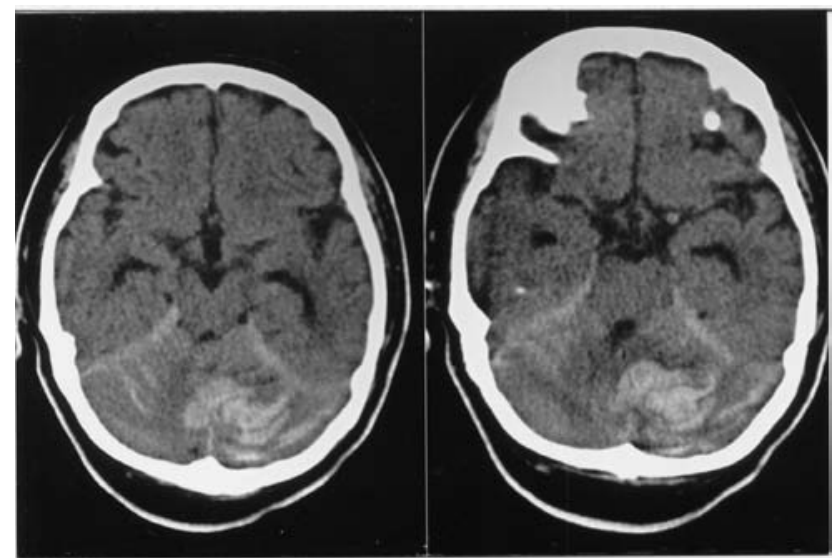

Figura 1. Caso 1. Cortes axiales de la TC craneal, donde se observa un hematoma en hemisferio cerebeloso izquierdo con sangre subcortical, en la superficie superior del cerebelo y en las folias cerebelosas, adquiriendo una distribución transversal en el sentido de las mismas. Aunque había hidrocefalia, ésta era previa, debido a la patología de base. El acueducto de Silvio y el IV ventrículo están permeables.

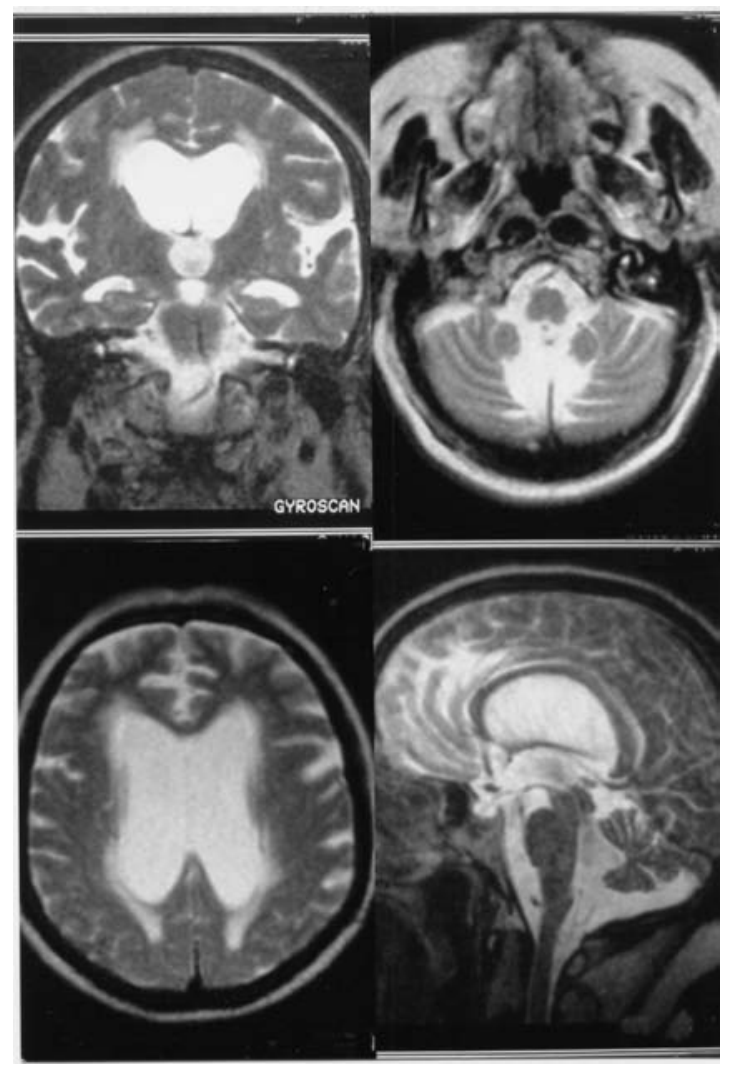

Figura 2. Caso 2. RM encefálica previa (cortes coronal, axiales y sagital en T2) que muestra la dilatación del sistema ventricular típica de la hidrocefalia crónica del adulto. No había imagen sugestiva de masa o malformación vascular en la fosa posterior.

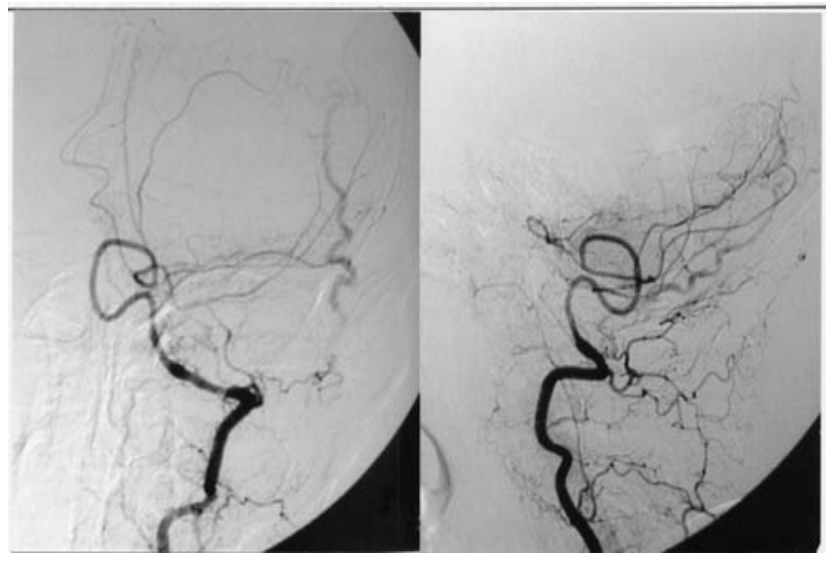

Figura 3. Caso 3. Cortes ántero-posterior y lateral de la angiografía de la arteria vertebral izquierda, que descartó patología vascular subyacente.

\section{Caso clínico 1}

Mujer de 64 años de edad con antecedentes personales de diabetes mellitus, hipertensión arterial y fibrilación auricular anticoagulada con acenocumarol. Vista en Consultas Externas por un cuadro clínico de imposibilidad para la deambulación e incontinencia urinaria ocasional, con afectación de funciones superiores y labilidad emocional. Los estudios de neuroimagen mostraron una hidrocefalia tetraventricular. Ante la sospecha de HCA se decidió a la colocación de un DLE como test diagnóstico. Tras sustituir el acenocumarol por heparina de bajo peso molecular unas semanas antes, se procedió a la colocación del DLE. En el traslado de la paciente desde quirófano a la planta de hospitalización, el drenaje se mantuvo accidentalmente abierto. A las 6 horas de su colocación, tras haber drenado $240 \mathrm{ml}$ de LCR, la paciente comenzó a presentar cefaleas, náuseas, vómitos y sudoración que inicialmente se achacaron a un cuadro de hipotensión intracraneal. En ningún momento se deterioró el nivel de conciencia. Ante la intensidad de los síntomas se realizó una TC craneal urgente que mostró la presencia de un hematoma en vermis y hemisferio cerebeloso izquierdo, con sangrado subaracnoideo en la superficie superior del cerebelo y en las folias cerebelosas (Figura 1). El DLE fue retirado inmediatamente y, dado el buen estado clínico de la paciente, se decidió seguir tratamiento conservador, con buena evolución clínica. Los estudios de neuroimagen (RM y angiografía cerebral) (Figuras 2 y 3 ) descartaron la existencia de patología subyacente.

La paciente se recuperó hasta su estado previo. Posteriormente le fue implantada una derivación ventrículoperitoneal, objetivándose gran mejoría clínica. 


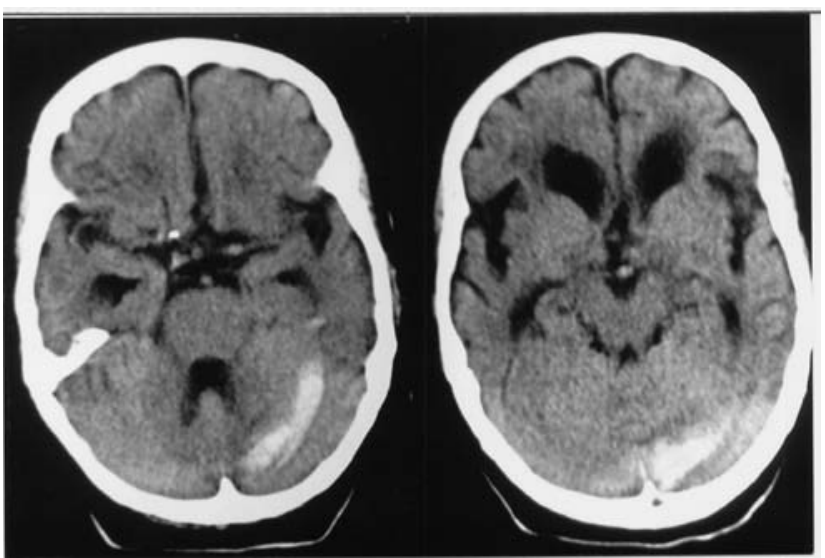

Figura 4. Caso2. Hematoma en el hemisferio cerebeloso izquierdo, siguiendo la orientación transversal de las folias cerebelosas. Se aprecia el escaso efecto de masa y que el IV ventrículo está permeable.

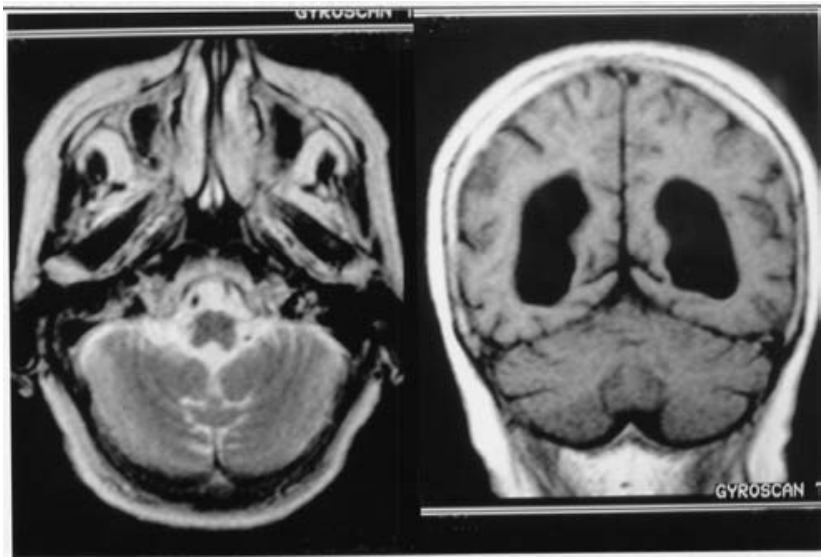

Figura 5. Caso 2. RM cerebral previa, corte axial en T2 y corte coronal en T1, que muestra la dilatación del sistema ventricular. No había patología vascular ni tumoral en el compartimento infratentorial.

\section{Caso clínico 2}

Mujer de 77 años de edad con único antecedente de hipertensión arterial. Presentaba un cuadro clínico consistente en ataxia de la marcha, incontinencia de esfínteres y trastorno de funciones superiores. Los estudios de neuroimagen mostraron dilatación del sistema ventricular, por lo que, ante la sospecha de una $\mathrm{HCA}$, se procedió a la colocación de un DLE. A las 30 horas de su colocación comenzó, bruscamente, a presentar cefaleas y vómitos no asociados a ortostatismo. No hubo deterioro del nivel de consciencia. En la bolsa de drenaje había $510 \mathrm{ml} \mathrm{de}$ LCR. Una TC craneal (Figura 4), mostró un hematoma en el hemisferio cerebeloso izquierdo, de disposición transversal, con escaso efecto de masa. Ante los hallazgos y la situación clínica de la paciente, se decidió la retirada del DLE y tratamiento conservador. En la RM cerebral realizada previa al ingreso (Figura 5) se había descartado patología subyacente. A los cinco días la paciente fue dada de alta con recuperación hasta su estado premórbido.

No se le implantó válvula de derivación al no objetivarse mejoría con la colocación de DLE.

\section{Discusión}

Los HCD han sido descritos como complicación de cirugía intracraneal $1^{4,6,8,10,12,16-18,22,23,27,31,34-38}$. Las intervenciones donde se describe con más frecuencia este tipo de complicación son la lobectomía temporal o frontal para el tratamiento de la epilepsia o la cirugía de aneurismas ${ }^{10,16,31,34}$. Ambas son situaciones en las que se produce una importante pérdida de LCR.

Los casos publicados tras cirugía espinal son menos numerosos ${ }^{2,7,9,11,13,19,21,25,26,30,33}$ (Tabla 1). En todos estos casos hubo pérdida de LCR en la cirugía, con apertura dural en los casos de cirugía extradural y con desarrollo de un hematoma de cerebelo dentro de las siguientes 120 horas del postoperatorio.

La crisis hipertensiva secundaria a hipertensión arterial se considera la causa más frecuente de los hematomas de cerebelo espontáneos ${ }^{15}$. Otros factores tales como las coagulopatías, el tratamiento anticoagulante, aneurismas o malformaciones arteriovenosas han sido identificados como posibles factores predisponentes. Sin embargo, en los HCD, la etiopatogenia no está del todo aclarada. La mayoría de los autores ${ }^{1,5,10,11,19,26,34}$ está de acuerdo en que el hiperdrenaje de LCR produce un descenso del cerebelo, con tracción sobre las venas puente, que sufrirían un estrechamiento y obstrucción de las mismas, produciéndose, en consecuencia, un infarto venoso hemorrágico.

Como exponen Brockmann et al..$^{5}$, parece existir un patrón característico de sangrado en los hematomas de cerebelo por pérdida de LCR. Primero, son sangrados de origen venoso, de localización en la parte superior del vermis y los surcos cerebelosos o folias, donde se localizan las venas puente del cerebelo. Segundo, suelen ser bilaterales y transversales, siguiendo el trayecto curvilíneo de las folias cerebelosas.

No hemos hallado en la literatura ningún caso de HCD como complicación de DLE. Sin embargo, consideramos que, desde el punto de vista etiopatogénico, los casos que aquí presentamos guardan una estrecha relación con los demás casos de hematomas cerebelosos tras cirugía intracraneal y, sobre todo, intrarraquídea, al ser el drenaje de LCR el factor más probablemente involucrado. Así mismo, las características radiológicas de ambos casos son similares a las que describen Brockmann et al. ${ }^{5}$ para los hematomas de cerebelo debidos a pérdida de LCR.

El síndrome de hipotensión de LCR es una complicación 


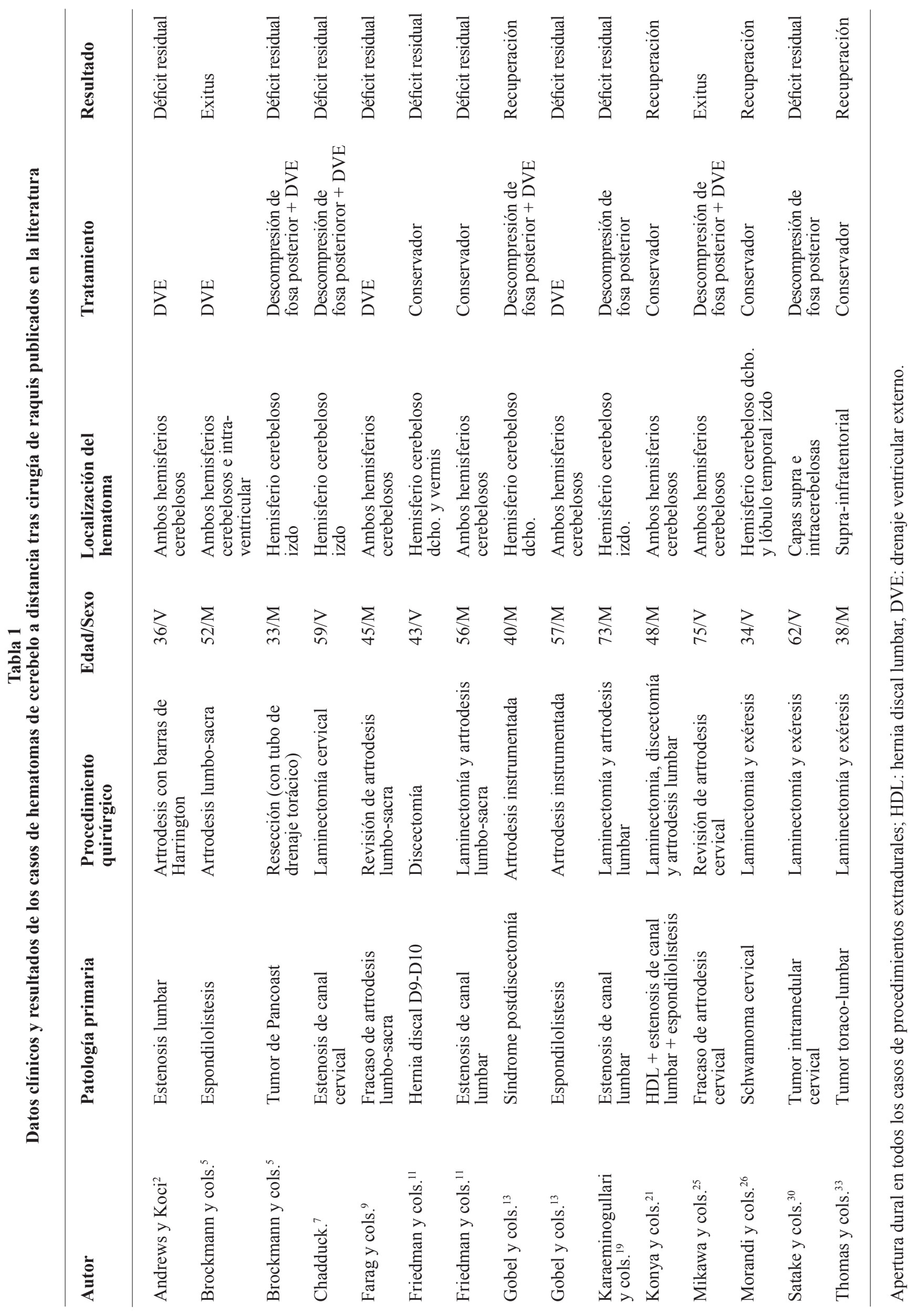


relativamente frecuente tras la colocación de un $\operatorname{DLE}^{24,28}$. Clínicamente suele cursar con cefaleas, que típicamente se alivian con el decúbito, náuseas y vómitos, sin alteración del nivel de consciencia. Pero si tras la colocación de un DLE el síndrome es muy acusado y/o va acompañado de alteración del nivel de consciencia, signos de disfunción cerebelosa o de tronco, es mandatorio realizar una TC craneal urgente para descartar la existencia de un hematoma a distancia.

El tratamiento de los HCD, al igual que aquéllos de otra etiología, dependerá del estado clínico del paciente y de las características radiológicas del hematoma, pudiendo mantenerse una actitud conservadora en los casos en que mantengan buen nivel de consciencia y sin signos de compresión, como en nuestros casos, aunque en ocasiones puede ser necesario el tratamiento quirúrgico. De cualquier forma creemos que la primera medida a adoptar tras el diagnóstico de un HCD debería ser el cierre o retirada del DLE.

En los casos que presentamos la evolución fue satisfactoria, sin presentar secuelas neurológicas. Sin embargo, Konya et al., en una revisión de $10 \mathrm{HCD}$ tras cirugía espinal ${ }^{21}$, refieren 1 fallecimiento, y 6 casos en los que los pacientes quedaron con algún tipo de secuela. En una revisión de 42 casos de HCD tras cirugía craneal que hace Friedman et al. ${ }^{10}$, refieren 2 fallecimientos y persistencia de secuelas en otros 11 pacientes.

\section{Conclusión}

El desarrollo de un hematoma de cerebelo, aunque muy raro, debe ser considerado entre las complicaciones de un DLE. Clínicamente puede cursar como un síndrome de hipotensión de LCR, si bien es más intenso y no cede con el decúbito. La realización de una TC urgente confirmará tal posibilidad, permitiendo adoptar las medidas terapéuticas más apropiadas. Para los casos en que el DLE se use como test diagnóstico de la HCA, hay que estar muy atentos a su manejo para evitar el hiperdrenaje de LCR.

\section{Bibliografía}

1. Acikbas, S.C., Akyuz, M., Kazan, S., Tuncer, R.: Complications of closed continuous lumbar drainage of cerebrospinal fluid. Acta Neurochir (Wien) 2002 May; 144: 475-80.

2. Andrews, R.T., Koci, T.M.: Cerebellar herniation and infarction as a complication of an occult postoperative lumbar dural defect. AJNR 1995; 16: 1312-1315.

3. Bloch, J., Regli, L.: Brain stem and cerebellar dysfunction after lumbar spinal fluid drainage. Case report. J Neurol Neurosurg Psychiatr 2003; 74: 992-994.

4. Brisman, M.H., Bederson, J.B., Sen, C.N., Germano, I.M., Moore, F., Post, K.: Intracerebral hemorrhage occurring remote from the craniotomy site. Neurosurgery 1996; 39: 1114-1122.

5. Brockmann, M.A., Nowak, G., Reusche, E., Russlies, M., Petersen, D.: Zebra sign: cerebellar bleeding pattern characterisitc of cerebrospinal fluid loss. J Neurosurg 2005; 102 : 1159-1162.

6. Cloft, H.J., Matsumoto, J.A., Lanzino, G., Cail, W.S.: Posterior fossa hemorrhage after supratentorial surgery. AJNR 1997; 18: 1573-1580.

7. Chadduck, W.M.: Cerebellar hemorrhage complicating cervical laminectomy. Neurosurgery 1981; 9: 185-189.

8. de Paola, L., Troiano, A.R., Germiniani, F. et al.: Cerebellar hemorrhage as a complication of temporal lobectomy for refractory medial temporal epilepsy. Report of three cases. Arq Neuropsiquiatr 2004; 62: 519-522.

9. Farag, E., Abdou, A., RIAD, I., Borsellino, S.R., Schubert, A.: Cerebellar hemorrhage caused by cerebrospinal fluid leak after spine surgery. Anesth Analg 2005; 100: 545-546.

10. Friedman, J.A., Piepgras, D.G., Duke, D.A., et al.: Remote cerebellar hemorrhage after supratentorial surgery. Neurosurgery 2001; 49: 1327-1340.

11. Friedman, J.A., Ecker, R.D., Piepgras, D.G., Duke, D.A.: Cerebellar hemorrhage after spinal surgery: report of two cases and literature review. Neurosurgery 2002; 50:13611364.

12. Gelfenbeyn, M., Vasilev, S., Krylov, V.: Cerebellar hemorrhage after supratentorial aneurysm surgery with lumbar drainage. Neurosurg Rev 2001; 24: 214-219.

13. Gobel, F., Heidecke, V., Hube, R., Reichel, H., Held, A., Hein, W.: Cerebellar hemorrhage as an early complication of spinal operations. Two case reports and review of the literature. Z Orthop Ihre Grenzgeb 1999; 137: 371-375.

14. Graf, C., Gross, C., Bech, D.: Complications of spinal drainage in the management of cerebrospinal fluid fstula. J Neurosurg 1981; 34: 392-395.

15. Heiskanen. O.: Treatment of spontaneous intracerebral and intracerebellar hemorrhages. Stroke 1993 Dec; 24 (12 Suppl): 194-195.

16. Honegger, J., Zentner, J., Spreer, J., Carmona, H., Schulze-Bonhage, A.: Cerebellar hemorrhage arising postoperatively as a complication of supratentorial surgery: a retrospective study. J Neurosurg 2002; 96: 248-254.

17. Hyam, J.A., Turner, J., Peterson, D.: Cerebellar haemorrhage after repeated burr hole evacuation for chronic subdural haematoma. J Clin Neurosci 2007 Jan; 14: 83-86.

18. Kaplan, S.S., Lauryssen, C.: Cerebellar haemorrhage after evacuation of an acute supratentorial subdural haematoma. British Journal of Neurosurgery 1999; 13: 329-331.

19. Karaeminogullari, O., Atalay, B., Sahin, O., et al.: Remote cerebellar hemorrhage alter a spinal surgery complicated by dural tear: case report and literature review. Neurosurgery 57 [ONS Suppl 1]: ONS-215, 2005.

20. Kitchel, S., Eismont, F., Green, B.: Closed subarach- 
noid drainage for management of cerebrospinal fluid leakage after an operation on the spine. J Bone Joint Surg 1989; 71A: 984-987.

21. Konya, D., Ozgen, S., Pamir, M. N.: Cerebellar hemorrhage after spinal surgery: case report and review of the literature. Eur Spine J 2006; 15: 95-99.

22. Marquardt, G., Setter, M., Schick, U., Seifer, V.: Cerebellar hemorrhage after supratentorial craniotomy. Surg Neurol 2002; 57: 241-252.

23. Maruyama, T., Ishii, K., Isono M., Abe, T., Fujiki, M., Kobayashi, H.: Remote cerebellar hemorrhage following supratentorial craniotomy. Case report. Neurol Med Chir 2004; 44: 294-297.

24. McCallum, J., Maroon, J., Janetta, P.: Treatment of postoperative cerebrospinal fluid fistulas by subarachnoid drainage. J Neurosurg 1975; 43: 434-437.

25. Mikawa, Y., Watanabe, R., Hino, Y., Ishii, R., Hirano, K.: Cerebellar hemorrhage complicating cervical durotomy and revision C1-C2 fusion. Spine 1994; 19: 1169-1171.

26. Morandi, X., Riffaud, L., Carsin-Nicol, B., Guegan, Y.: Intracerebral hemorrhage complicating cervical "hourglass" schwannoma removal. J Neurosurg 2001; 94: 150-153.

27. Papanastassiou, V., Kerr, R., Adams, Ch.: Contralateral cerebellar hemorrhagic infarction after pterional craniotomy: report of five cases and review of the literature. Neurosurgery 1996; 39: 841-852.

28. Roland, P.S., Marple, B.F., Meyerhoff, W.L, Mickey, B.: Complications of lumbar spinal fluid drainage. Otolaryngol Head Neck Surg 1992; 107: 564-69.

29. Ruiz Sandoval, J.L., Campos, A., Romero-Vargas, S., Jiménez-Rodríguez, M.I., Chiquete, E.: Multiple simultaneous intracerebral hemorrhages following accidental massive lumbar cerebrospinal fluid drainage: case report and literature review. Neurol India 2006 Dec; 54: 421-424.

30. Satake, K., Matsuyama, Y., Iwata, H., Sato, K., Kawakami, N: Cerebellar hemorrhage complicating resection of a cervical intramedullary tumour. Spinal Cord 2000; Aug: 38: 504 .
31. Siu, T.L.T., Chandran, K.N.: Cerebellar hemorrhage following supratentorial craniotomy. Journal of Clinical Neuroscience 2003; 10: 378-384.

32. Snow, R.B., Kuhel, W., Martin, S.B.: Prolonged lumbar spinal drainage after the resection of tumors of the skull base: a cautionary note. Neurosurgery 1991 Jun; 28: 880-882.

33. Thomas, G., Jayaram, H., Cudlip, S., Powell, M.: Supratentorial and infratentorial intraparenchymal hemorrhage secondary to intracranial CSF hypotension following spinal surgery. Spine 2002; 27: E410-E412.

34. Toczek, M.T., Morrell, M.J., Silverberg, G.A., Lowe, G.M.: Cerebellar hemorrhage complicating temporal lobectomy. J Neurosurg 1996; 85: 718-722.

35. Tomii, M., Nakajima, M., Ikeuchi, S., Ogawa, T., Abe, T.: Infratentorial hemorrhage following supratentorial surgery. No Shinkei Geka 1999 Oct; 27: 921-925. (Abstract).

36. van Calenberg, F., Goffin, J., Plets, C.: Cerebellar hemorrhage complicating supratentorial craniotomy: report of two cases. Surg Neurol 1993; 40: 336-338.

37. Vogels, R.L., Verstegen, M.J., van Furth, W.R.: Cerebellar haemorrhage after non-traumatic evacuation of supratentorial chronic subdural haematoma: report of two cases. Acta Neurochir (Wien) 2006 Sep; 148: 993-996.

38. Yacubian, E.M., de Andrade, M.M., Jorge, C.L., Valerio, R.M.: Cerebellar hemorrhage after supratentorial surgery for treatment of epilepsy: report of three cases. Neurosurgery 1999; 45:159-165.

Bernal-García L.M.; Cabezudo-Artero J.M.; Ortega-Martínez, M.; Fernández-Portales I.; Ugarriza-Echebarrieta L.F.; Pineda-Palomo M.; Porras-Estrada L.F.; GómezPerals L.F.: Hematomas de cerebelo como complicación de drenaje lumbar. Presentación de dos casos y revisión de la literatura. Neurocirugía 2008. 19: 440-445.

Correspondencia postal: Dr. Luis Miguel Bernal García. S. de Neurocirugía. Hospital Infanta Cristina. Avenida de Elvas s/n. Badajoz. 